\title{
Aula Invertida: Percepciones de Docentes y Alumnos.
}

\section{Flipped Classroom: Perceptions of teachers and students}

Presentación: 11/10/2020

Aprobación: 21/12/2020

\section{María del Carmen Maurel}

Universidad Tecnológica Nacional -Facultad Regional Resistencia-Argentina

delcarmenmaurel@gmail.com.ar

\section{Albarenga, Joaquín Hernán \\ Universidad Tecnológica Nacional -Facultad Regional Resistencia-Argentina joalbarenga@gmail.com}

\section{Teruel Alonso, Melina Anahí}

Universidad Tecnológica Nacional -Facultad Regional Resistencia-Argentina meli.a.teruel@gmail.com

\section{Resumen}

La metodología de clase invertida es una de las opciones que actualmente se utiliza para mejorar el rendimiento académico de los alumnos y mantener la motivación en las actividades propuestas. El trabajo presenta los resultados obtenidos en una encuesta a docentes y a alumnos que permite conocer la percepción que los mismos tienen sobre la aplicación de dicha metodología y si la implementación de ésta permite la generación de competencias. Se obtuvieron resultados referidos principalmente al conocimiento de la metodología por parte de alumnos y docentes: ambos actores conocen de la metodología, los docentes están dispuestos a capacitarse sobre nuevas herramientas para poder llevar a cabo la implementación o mejorarla. Se aprecia una disparidad en reconocer que la adopción de la clase invertida puede propiciar la generación de competencias, en este punto debería verificarse que se esté implementando correctamente la metodología y que los alumnos conozcan la forma que se llevará a cabo ; además se deberá indagar qué otras variables pueden afectar esta discrepancia.

Palabras claves: clase invertida, percepciones, competencias, ingeniería

\footnotetext{
Abstract

The flipped classroom methodology is one option currently used to improve students' academic performance and keep the motivation in the proposed activities. The work presents
} 
the results obtained in a survey of teachers and students that allows to know the perception that they have about the application of the methodology and if its implementation allows the generation of skills. Mainly can be mentioned as results that both students and teachers know about the methodology, teachers are willing to be trained on new tools in order to carry out the implementation or improve it. There is a disparity in recognizing that the adoption of the flipped classroom can promote the generation of competences, it should be verified: the correct implementation of the methodology and the students' knowledge of how to carry it out

Keyword: flipped classroom - perceptions- skills - engineering

\section{Introducción.}

En la búsqueda de mejorar el rendimiento académico de los alumnos, mantener la motivación en las actividades propuestas y, recientemente, la generación de competencias se viene incorporando el uso de las TIC como complemento (en un primer momento) a las clases tradicionales, luego como herramientas que propician el buen rendimiento de los alumnos y el involucramiento de estos. Ante estas iniciativas, el rol tanto del alumno como del profesor han ido cambiando, tanto es así que el alumno pasa a tener una participación más activa y el profesor actúa de tutor más que de expositor. Actualmente, se han realizado numerosas experiencias en el modelo que se denomina "Aula invertida" o "Clase invertida" o "Flipped Classroom". Una de las propuestas de este nuevo modelo, es el uso de tecnología multimedia para que los alumnos - antes de la clase - puedan incorporar los temas que serán luego desarrollados de manera más práctica en la clase presencial.

En la actualidad, se encuentran varios trabajos relacionados con la temática, como el estudio realizado por (Rodríguez, 2016); donde se busca comprender la perspectiva de los participantes sobre el modelo de clase invertida. Entre los principales resultados se evidenció una percepción positiva de la aplicación del modelo, y la comprensión de las tareas tanto extraclase como interclase; en este sentido se considera relevante la correcta comunicación, la orientación del docente, el trabajo autónomo y colaborativo.

En el trabajo realizado por (Berenguer et al., 2016) se pretende analizar la utilidad del modelo pedagógico y de los diversos medios (videos, podcast, etc) para llevarlo a la práctica. Entre las conclusiones que llega la autora es que el éxito del modelo requiere que el alumno se involucre $100 \%$ en el proceso de aprendizaje, ya que se requiere que el alumno trabaje por sí mismo y fuera del aula para la comprensión de los conceptos teóricos, y el tiempo de clase se aproveche para resolver dudas que surgieron al visualizar el material, realizar prácticas y propiciar la discusión en el aula. Luego, resulta importante la capacidad del profesor de generar los recursos adecuados para poder llevar a cabo la actividad, donde la planificación de las actividades antes, durante y después del aula deben estar correctamente sincronizadas para poder lograr los objetivos que se pretenden con la aplicación de esta metodología.

En el trabajo de (Al Jassmi et al., 2019); se plantea la implementación de la clase invertida en clases de la carrera de Ingeniería Civil. En esta propuesta se pretende analizar la percepción que los alumnos tienen sobre esta nueva metodología. Se definió una secuencia de trabajo que consiste en: diseñar la clase invertida en donde se crea por ejemplo el video que utilizarán los alumnos, previo a la clase; y la definición de las actividades que se desarrollarán en la clase; implementación de la clase invertida: se envía el video a los alumnos con 
las instrucciones para verlo antes de clases y una guía de las actividades que se realizarán; "tiempo de calidad" llaman así a la puesta en común realiza en el aula y la actividades de resolución de problemas; la última instancia "tiempo de calidad de encuesta y reflexión" aquí se propone a los alumnos que luego del horario de clase respondan una encuesta para volcar sus opiniones acerca de la metodología implementada. Los resultados que se obtuvieron permitieron obtener algunas conclusiones tales como: los estudiantes que implementaron la estrategia de aula invertida obtuvieron mejores resultados en la resolución de los problemas; algunos alumnos tenían dudas sobre esta forma de implementación pero se adecuaron rápidamente a la nueva metodología; una sugerencia interesante que se debe tener en cuenta es que proponen tomar 15 o 20 minutos de la clase antes de la resolución de los ejercicios para realizar una recopilación de los temas del video, con el fin de aclarar algunos conceptos que pudieran no haber quedado claro.

Actualmente en Argentina, en la enseñanza de la Ingeniería; se está trabajando en la generación de competencias en los alumnos. El CONFEDI (Consejo federal de decanos de ingeniería) decidió trabajar en la segunda generación de estándares para la acreditación de carreras de Ingeniería, generando así el Libro Rojo (Ingeniería, 2018). Los nuevos estándares de acreditación aquí propuestos se aplicarán a todas las carreras de Ingeniería, entre las cuales se encuentra la terminal Ingeniería en sistemas de Información. Estos estándares están definidos en términos de formación por competencias. En este sentido es necesario aclarar qué entendemos por competencia: es la capacidad de articular eficazmente un conjunto de esquemas (estructuras mentales) y valores, permitiendo movilizar (poner a disposición) distintos saberes, en un determinado contexto con el fin de resolver situaciones profesionales. Esta definición señala que las competencias:

- aluden a capacidades complejas e integradas

- están relacionadas con saberes (teórico, contextual y procedimental),

- se vinculan con el saber hacer (formalizado, empírico, relacional)

- están referidas al contexto profesional (entendido como la situación en que el profesional debe desempeñarse o ejercer)

- están referidas al desempeño profesional que se pretende (entendido como la manera en que actúa un profesional técnicamente competente y socialmente comprometido)

- incorporan la ética y los valores

Ahora bien, si tenemos en cuenta que buscamos formar a los futuros ingenieros por competencias, que contamos con las TIC que están incorporadas al aula, y que la aplicación del aula invertida tiene entre sus objetivos el trabajo autónomo, colaborativo, buscar una actitud analítica y crítica en el alumno; entonces es menester un trabajo que pretenda analizar si el modelo de clase invertida propicia la generación de competencias en los alumnos de Ingeniería en Sistemas de Información. En este sentido se buscó evidencias al respecto.

En tal sentido en el artículo "Enseñanza de la ingeniería de software basada en competencias fundamentales y aula invertida", de (Bedoya et al, 2019) es una experiencia realizada en un curso de pregrado de los cursos de Ingeniería de Software de la Universidad de Caldas. Las fases generales de la propuesta son: Identificar competencias, clasificar competencias y definir estrategias de enseñanza. En la primera fase de la identificación de competencias, se determinaron aquellas competencias fundamentales de la disciplina, a partir de la cual se creó un mapa conceptual de saberes y prácticas. En la segunda, se clasificaron las 
competencias agrupadas por elementos comunes y separando aquellas que se consideraron demasiado amplias. En cuanto, a la definición de la estrategia de enseñanza se creó una guía o guión general para cada semana, donde se especifica las competencias y las prácticas que los alumnos deberán realizar. También se especifica la elaboración de los materiales educativos, en su mayoría se utilizan videos y presentaciones que son subidas al canal de youtube, pero que los alumnos deben visualizarlos desde la plataforma Edpuzzle. Esta plataforma permite realizar un seguimiento de los alumnos, quienes lo vieron, quienes respondieron las preguntas, etc. Durante las clases presenciales se desarrollan talleres guiados por el profesor utilizando diferentes técnicas de presentación. Como conclusiones los autores mencionan que ha permitido revisar y actualizar el plan de los cursos, desarrollar material adecuado y orientado a las nuevas generaciones, y la realización de actividades más prácticas y colaborativas lo que permite la apropiación de las competencias deseadas.

A nivel nacional, se ha consultado la experiencia desarrollada por (Ponce et al., 2017), donde se lleva a cabo la implementación de la clase invertida en el curso de Ambientación a la vida universitaria -módulo matemática destinados a ingresantes a la Facultad de Ciencias Agropecuarias de la Universidad Nacional de Entre Ríos. Para el desarrollo de la experiencia se realizó una "guía de estudio". Cada semana contaba con los contenidos teóricos que deberían desarrollarse, agregando a este material recursos audiovisuales y multimediales que son subidos al Aula virtual de la asignatura. Luego, en las clases presenciales los alumnos trabajaban en sesiones colaborativas de resolución de problemas y actividades de transferencia a situaciones cercanas a las Ciencias Agropecuarias. Los docentes durante las clases realizaron breves explicaciones sobre aspectos relevantes del contenido, algunas recomendaciones sobre errores habituales e indicaciones generales. Los autores realizan un análisis comparativo de resultados obtenidos con cortes anteriores, donde concluyen que encuentran varias ventajas en la aplicación de este modelo, entre las que se mencionan: la posibilidad de lograr un aprendizaje más profundo, la adquisición de competencias transversales y el aumento de la motivación del estudiante

La metodología es cuanti y cualitativa propuesta parte de los principios y herramientas que combina procedimientos de obtención de información y de análisis cualitativos y cuantitativos. Se plantea un estudio descriptivo, se busca especificar las características que adopta el modelo de clase invertida con la incorporación de las herramientas de la Web 3.0 en la carrera de Ingeniería en Sistemas de Información de la UTN FRRe.

Se pretende describir y relacionar la aplicación de clase invertida con la generación de ciertas competencias en los estudiantes; a través del uso de las herramientas de la web 3.0; el método a utilizar es correlacional. Pero también se busca comprender las características que asumen las diferentes utilizaciones de las herramientas; si logran constituirse en formatos de clases invertidas; cuál es la percepción que tienen del impacto, alumnos y docentes, sobre el modelo; por ello, es también un estudio de tipo etnográfico.

\section{Desarrollo}

El objeto que se propone este estudio es, un análisis de la utilización del modelo de clase invertida a través de las herramientas 3.0 para generar competencias de egreso en los estudiantes. El estudio se desarrolla en las cátedras de la carrera de Ingeniería en Sistema de Información. Para ello definimos una muestra representativa en cada nivel de la carrera. La muestra se compone por las siguientes cátedras: Algoritmo y estructura de datos y Sistemas y organizaciones (primer nivel); Análisis de sistemas y Sistemas operativos (segundo nivel); Diseño de Sistemas e Ingeniería y Sociedad (tercer nivel); Redes de información y 
Metodologías Ágiles (cuarto nivel); Sistemas de Gestión (Quinto nivel).

En una primera etapa se analizó cómo se está trabajando en esas cátedras, si se está trabajando con herramientas de la web 3.0 y si se aplica el modelo de aula invertida. En una segunda etapa se incluirán mejoras en las cátedras (para los que ya están implementando aula invertida como para los que recién van a incorporar el modelo) y se realizará un seguimiento y mediciones a través de diferentes instrumentos. Posteriormente, en una tercera etapa; se realizará una propuesta de mejora para implementar en las mismas cátedras seleccionadas.

En esta primera etapa la indagación sobre el estado de situación y las percepciones de los sujetos se realizó a través de dos encuestas: una para los alumnos y otra para los docentes de las cátedras involucradas en la muestra. Si bien se intentó recoger similares aspectos para el análisis, los instrumentos se adecuaron a las características de ambos actores.

\section{Análisis de los resultados}

A continuación, se presentan los resultados recogidos a partir de las encuestas realizadas a docentes y alumnos que participaron de la muestra del presente estudio.

Sobre un total de 22 docentes y 159 alumnos encuestados, se recogieron los siguientes datos:

En la distribución de los docentes encuestados según sus cargos; cómo se puede observar en el gráfico 1, más del 50 \% son docentes que desarrollan teoría.

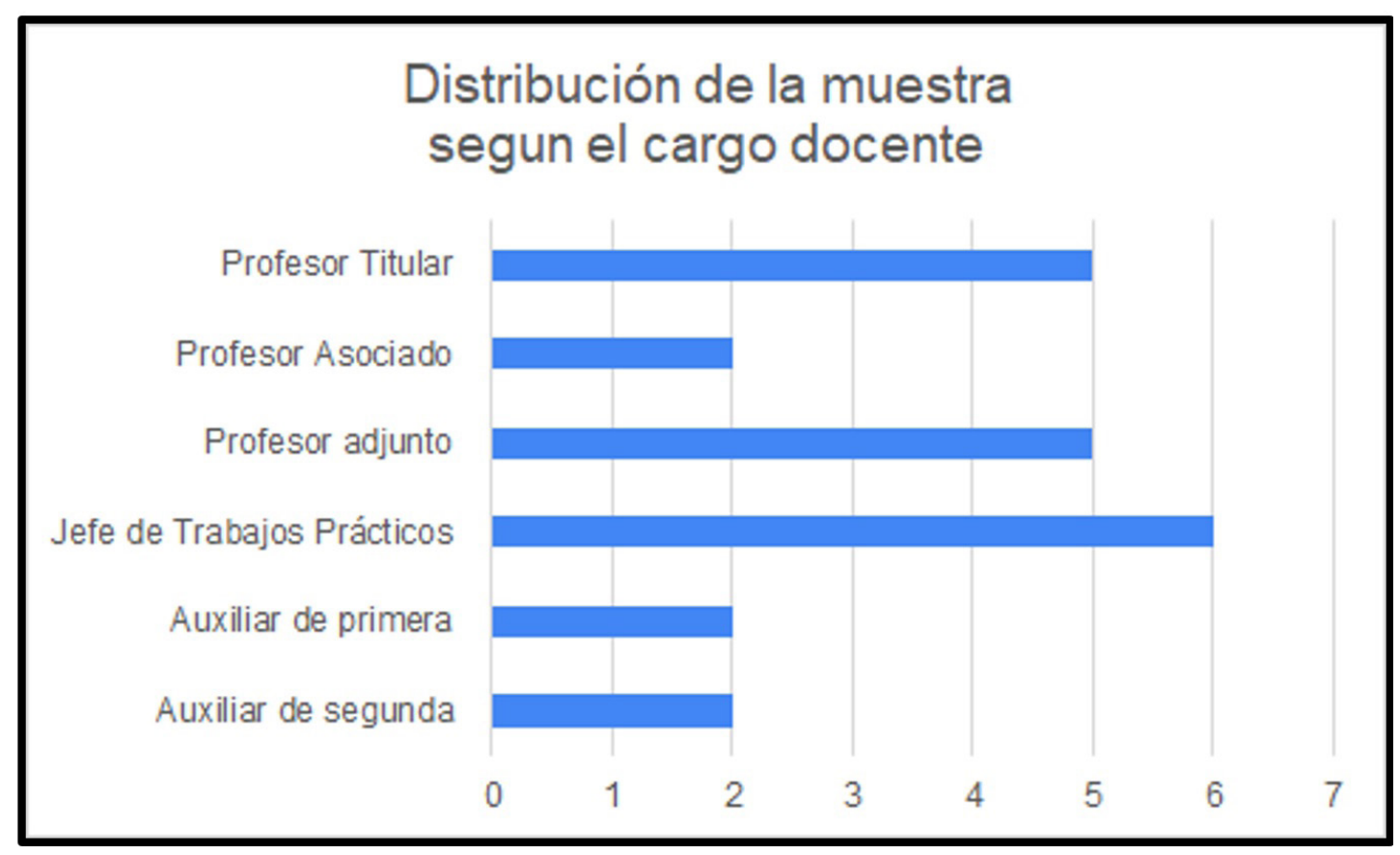

Gráfico N 1: Distribución de la muestra de docentes

En la distribución de los alumnos encuestados, y considerando el año que están cursando los alumnos que respondieron la encuesta, se puede observar que el mayor porcentaje se encuentra cursando el primer nivel $(36,5 \%)$ y el tercer nivel de la carrera $(35,8 \%)$, ver gráfico $\mathrm{N}^{\mathrm{o}} 2$. 


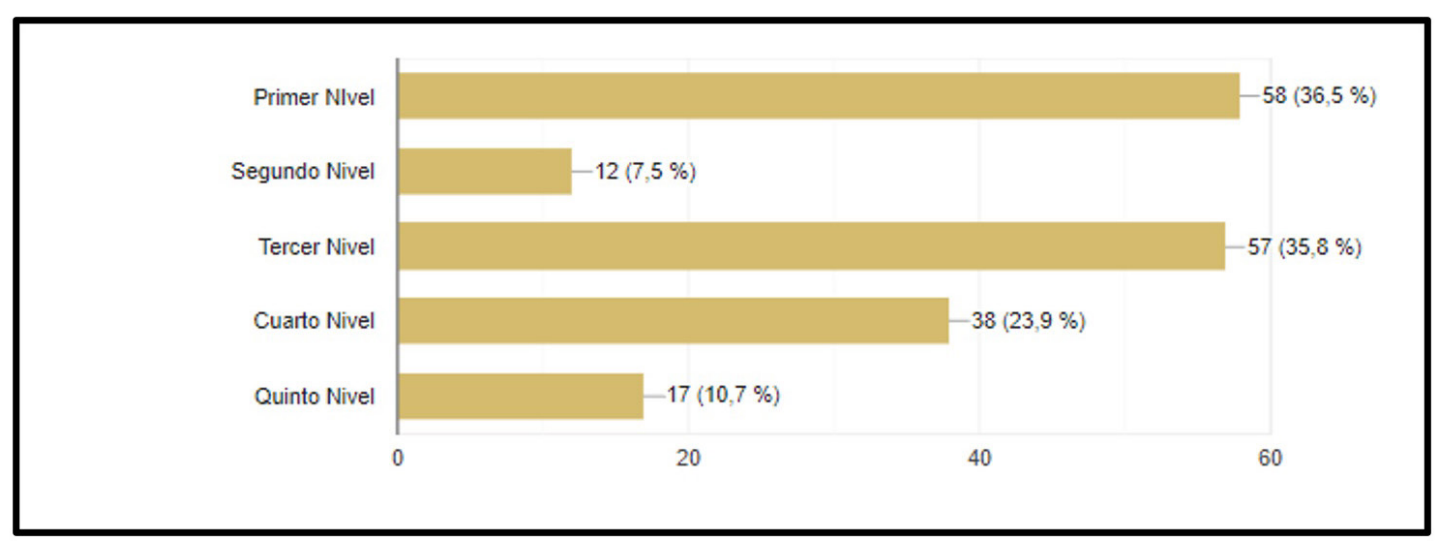

Gráfico N². Distribución de la muestra de alumnos según el nivel de cursada

En cuanto al conocimiento del concepto o metodología del aula invertida el $100 \%$ de los docentes contestaron afirmativamente, ver Gráfico $\mathrm{N}^{\circ} 3$.

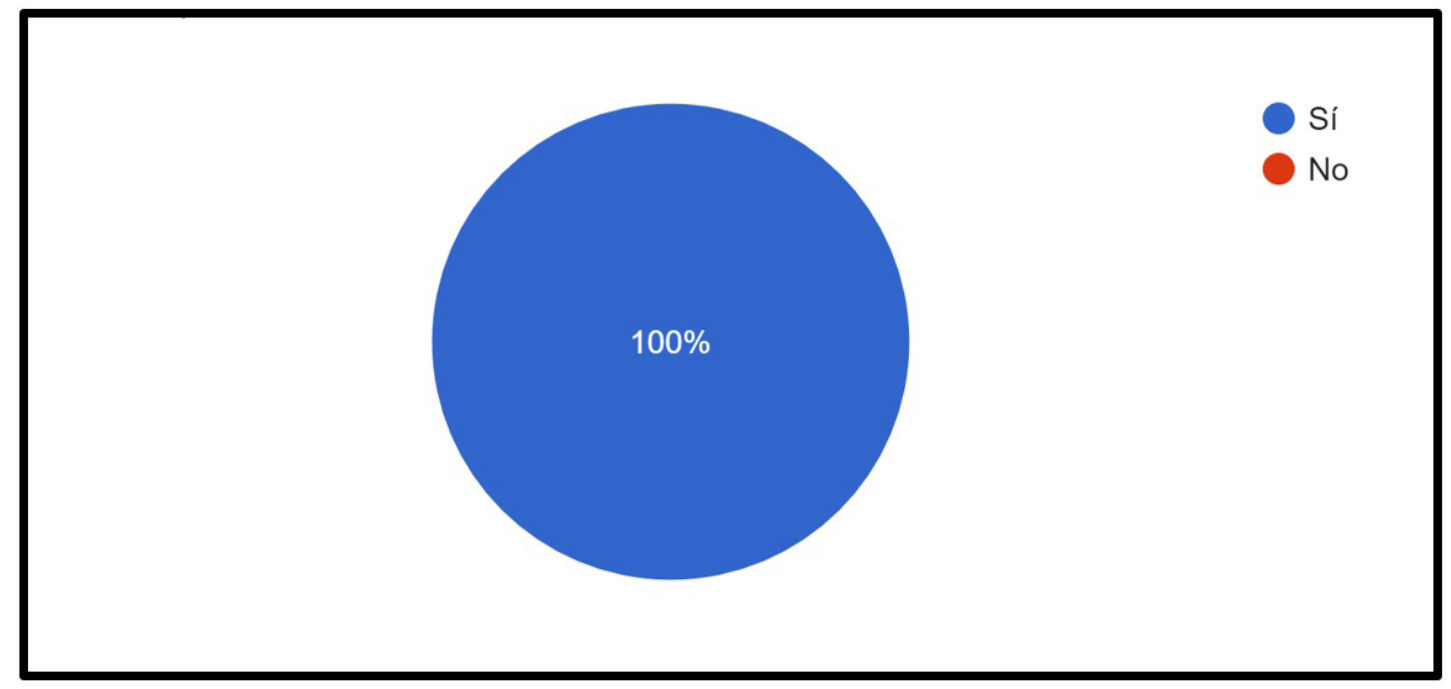

Gráfico №3: Conocimiento del concepto de aula invertida por parte de los docentes.

Mientras que los alumnos ante la misma pregunta, sólo el 66,7 \% manifestaron conocer el concepto (Gráfico $\left.\mathrm{N}^{\circ} 4\right)$ 


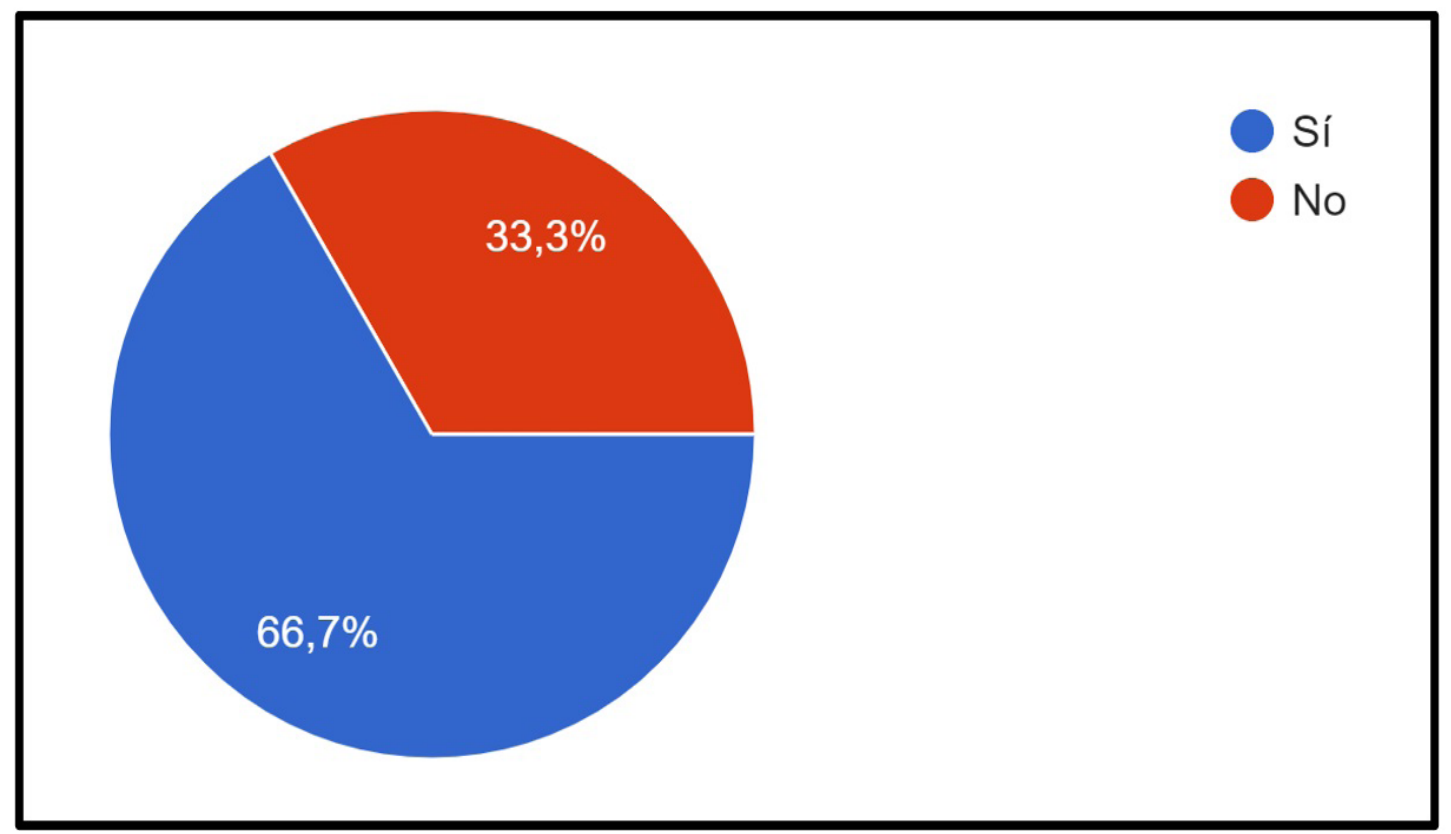

Gráfico $N^{\circ} 4$. Conocimiento sobre el aula invertida por parte de los alumnos.

Luego, sólo a los docentes se les dieron opciones relacionadas con el concepto de aula invertidas, donde los mismos debían seleccionar una opción que ellos consideran se acerca a lo que desarrollan en sus clases:

A. El Aula Invertida es un esquema en el que se busca mejorar los procesos de aprendizaje mediante la inversión de las actividades realizadas en el esquema tradicional de educación. En el Aula Invertida las clases son recibidas en casa a través de videos u otras herramientas tecnológicas y las tareas son realizadas en clase con acompañamiento del docente.

B. El Aula Invertida grupal es el modelo que agrega valor a la experiencia de aprendizaje a través de la interacción entre estudiantes. La clase se basa en videos u otros recursos que son digeridos antes de la clase. La diferencia es que los estudiantes deben formar equipos para trabajar juntos en los contenidos. Este formato anima a los estudiantes a aprender unos de otros. También refuerza habilidades blandas y el aprendizaje, ya que deben entender bien la temática para explicarla a sus pares.

C. El Aula Invertida Doble pone al estudiante en el rol del instructor. En este modelo los estudiantes graban sus propios videos para demostrar dominio y nuevas competencias. Nuevamente, el acto de mostrar o enseñar cómo se hace algo refuerza el aprendizaje.

Como se puede observar en el en el gráfico $\mathrm{N}^{\circ} 5$, la mayoría optó por la primera opción: la A. 


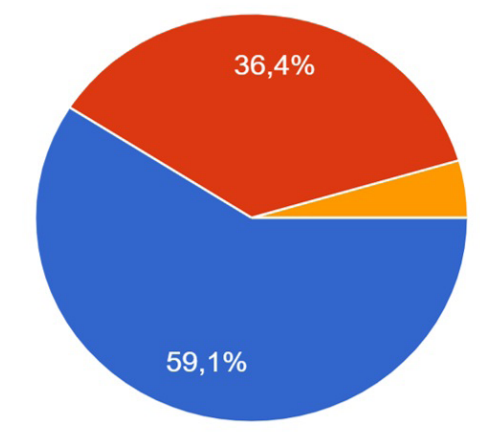

El Aula Invertida es un esquema en el que se busca mejorar los procesos de aprendizaje mediante la inversión de las actividades realizadas en el esquema...

El Aula Invertida grupal es el modelo que agrega valor a la experiencia de aprendizaje a través de la interacción entre estudiantes. La clase se basa e...

El Aula Invertida Doble pone al estudiante en el rol del instructor. En este modelo los estudiantes graban s...

Gráfico $\mathrm{N}^{\circ} 5$. Conceptos sobre aula invertida

Con relación a los tipos de aula invertida, se les explicó tanto a los docentes como a los alumnos, que se habla de 7 tipos de aula invertida, si bien no son los únicos. Luego se les solicitó seleccionen una o más opciones, que según ellos se ajustan más a un aula invertida.

Las opciones fueron las siguientes:

1. Clase invertida estándar. Los alumnos trabajan con vídeos en casa y practican lo aprendido con deberes tradicionales en el aula.

2. Clase inversa orientada al debate. Los vídeos sirven para desarrollar debates o reflexiones posteriores dentro del aula.

3. Clase inversa orientada a la experimentación. Los vídeos de los profesores youtubers sirven como referencia para recordar el aprendizaje de distintas materias.

4. Clase inversa como aproximación. Los estudiantes ven los vídeos en clase. Después el profesor resuelve sus dudas, recomendado para alumnos de menor edad.

5. Clase inversa basada en grupos. Combina el tipo de clase inversa como aproximación con la salvedad de que los alumnos se agrupan para trabajar en distintas tareas asignadas.

6. Clase inversa virtual. Los conceptos de tiempo y espacio se redefinen y el concepto de aula tradicional queda suplido por el de aula virtual.

7. Inversión del profesor. El profesor crea vídeos para establecer base de aprendizaje en los alumnos o para demostrar experiencias.

Como se puede observar en el Gráfico $\mathrm{N}^{\circ} 6$, las opciones más seleccionadas, por los docentes fueron la 1,2 y 5 . 


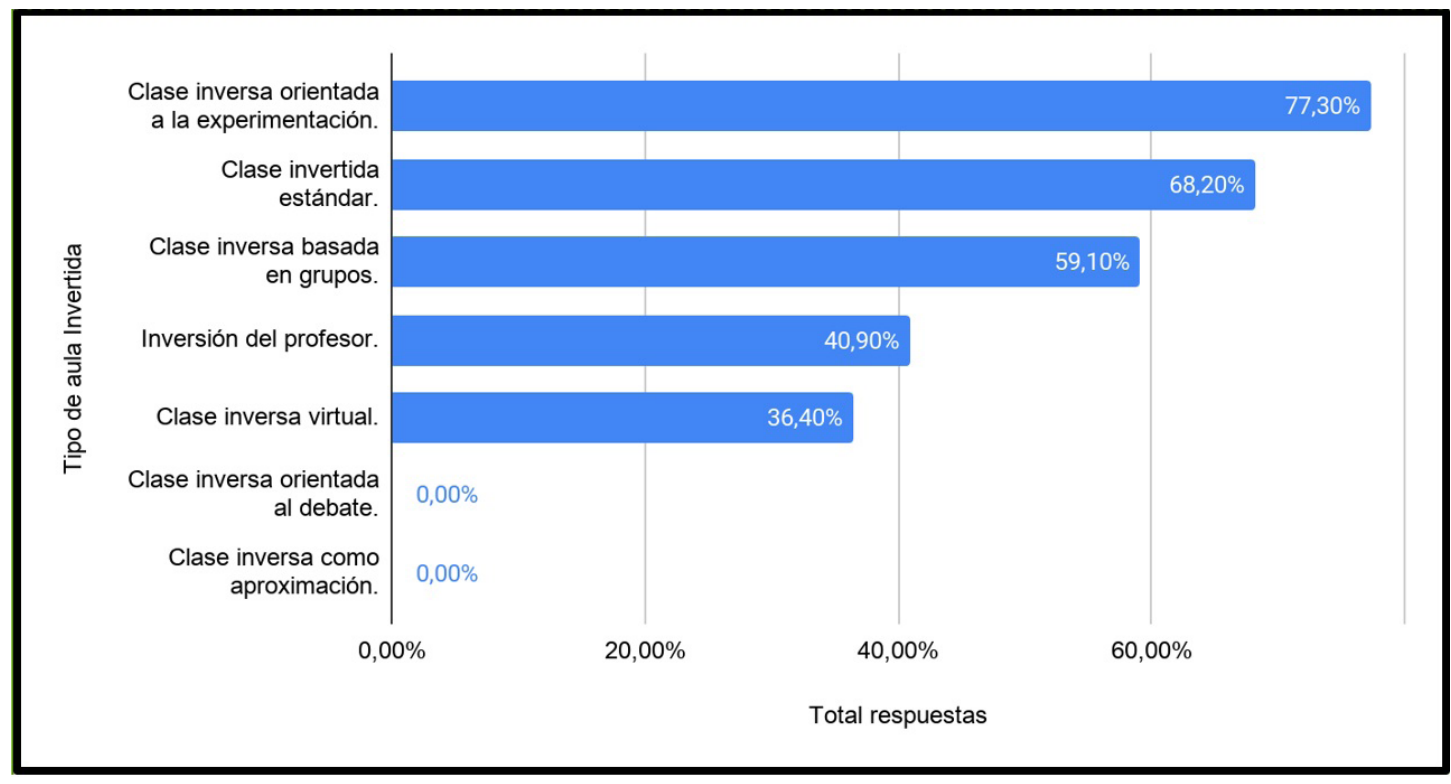

Gráfico N 6. Tipos de aula invertida-Docentes.

Sin embargo, en el caso de los alumnos se puede observar en el gráfico $\mathrm{N}^{\circ} 7$ que un 67,3 $\%$ seleccionó la primera opción, las otras están todas por debajo del $50 \%$. Se considera que esta opción es la más elegida porque de alguna forma es la experiencia vivida durante el cursado de la carrera.

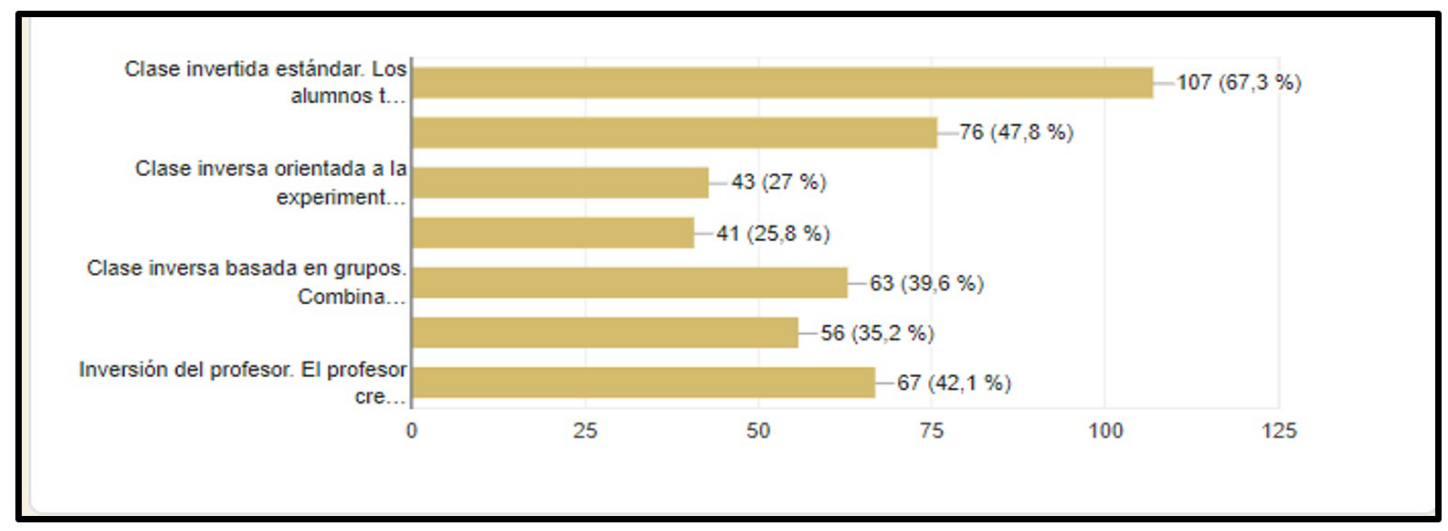

Gráfico № 7. Tipos de aula invertida-Alumnos.

Consultados sobre la adquisición de competencias a través del aula invertida, el $100 \%$ de los docentes afirmaron que es posible que el mismo contribuya a su adquisición. (Según se observa en el gráfico $\mathrm{N}^{\circ} 8$ ) 


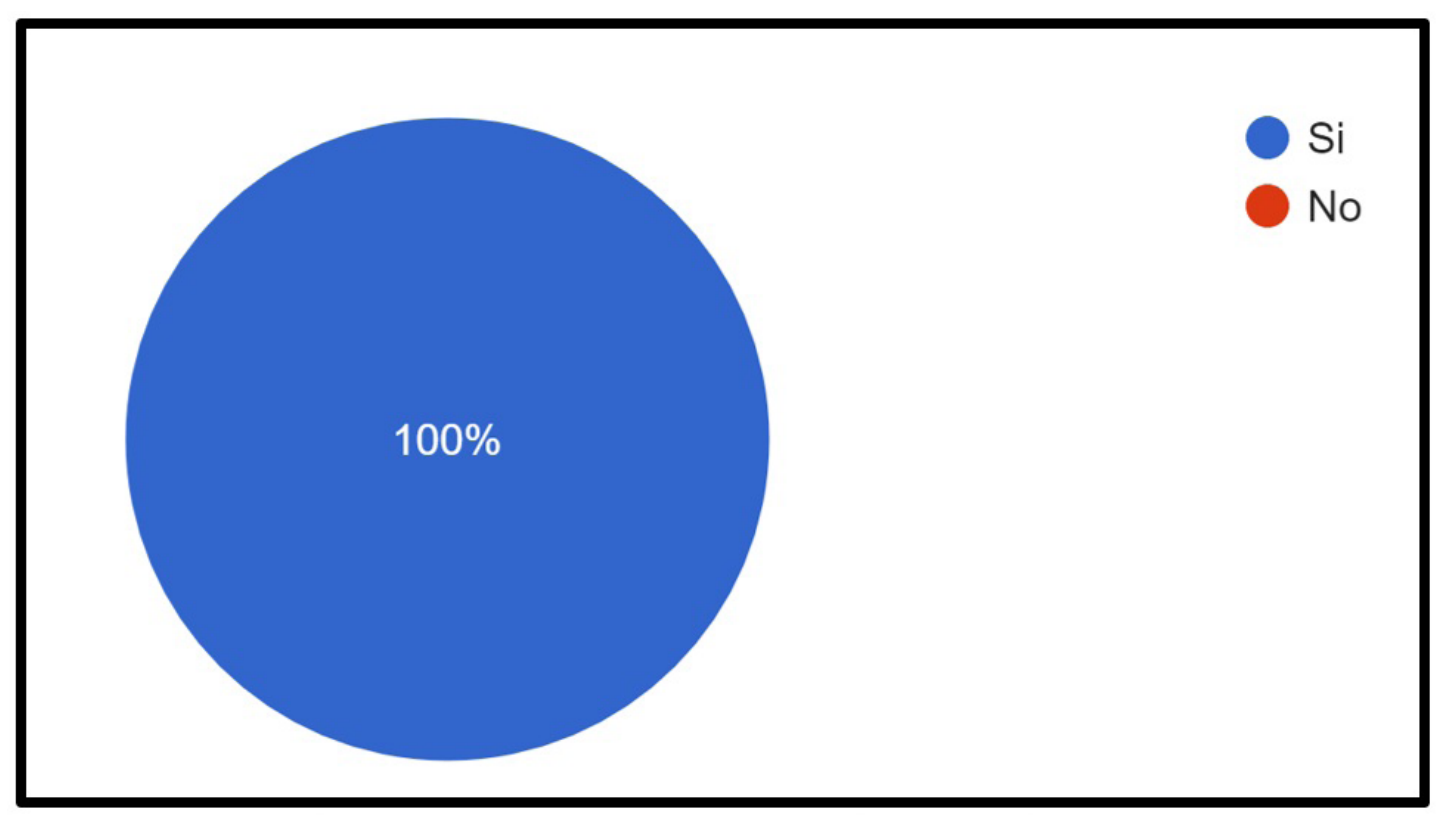

Gráfico Nº 8. Contribución del aula invertida en la formación de competencias-Docentes.

En tanto en el caso de los alumnos sólo un 76,7 \% considera que esta metodología contribuiría a la formación de competencias ingenieriles, ver gráfico $\mathrm{N}^{\circ} 9$.

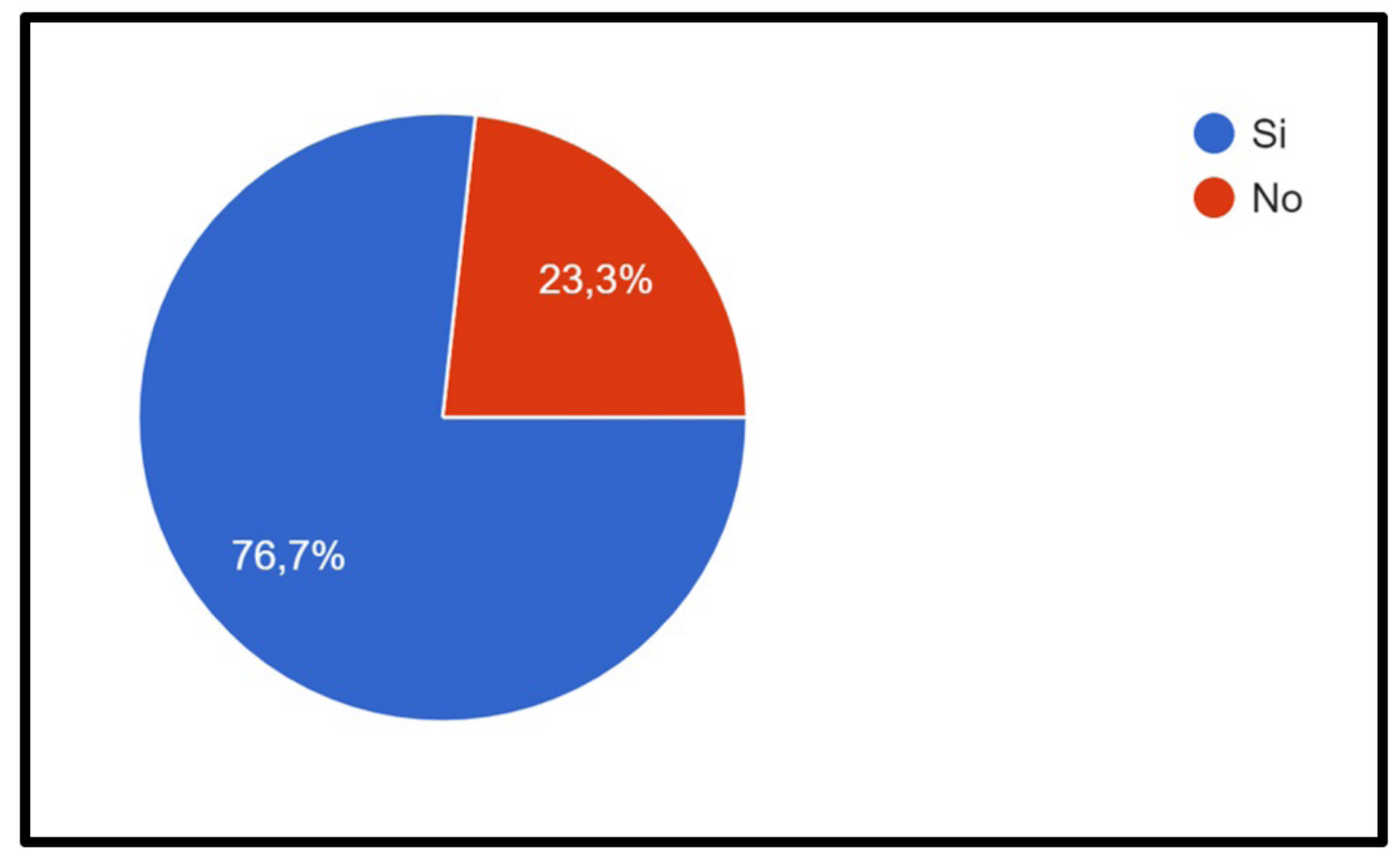

Gráfico № 9. Contribución del aula invertida en la formación de competencias-Alumnos. 
Ante la pregunta sobre cuáles serían las competencias más fáciles de lograr a través de la metodología de aula invertida, el $95 \%$ de los docentes, optó por la competencia de aprender en forma autónoma y de forma independiente; un $72 \%$ por la competencia relacionada con desempeñarse de manera efectiva en equipos de trabajo; y un $50 \%$ por la competencia de utilizar de manera efectiva las técnicas y herramientas de aplicación en la ingeniería. (gráfico $\mathrm{N}^{\mathrm{o}} 10$ ).

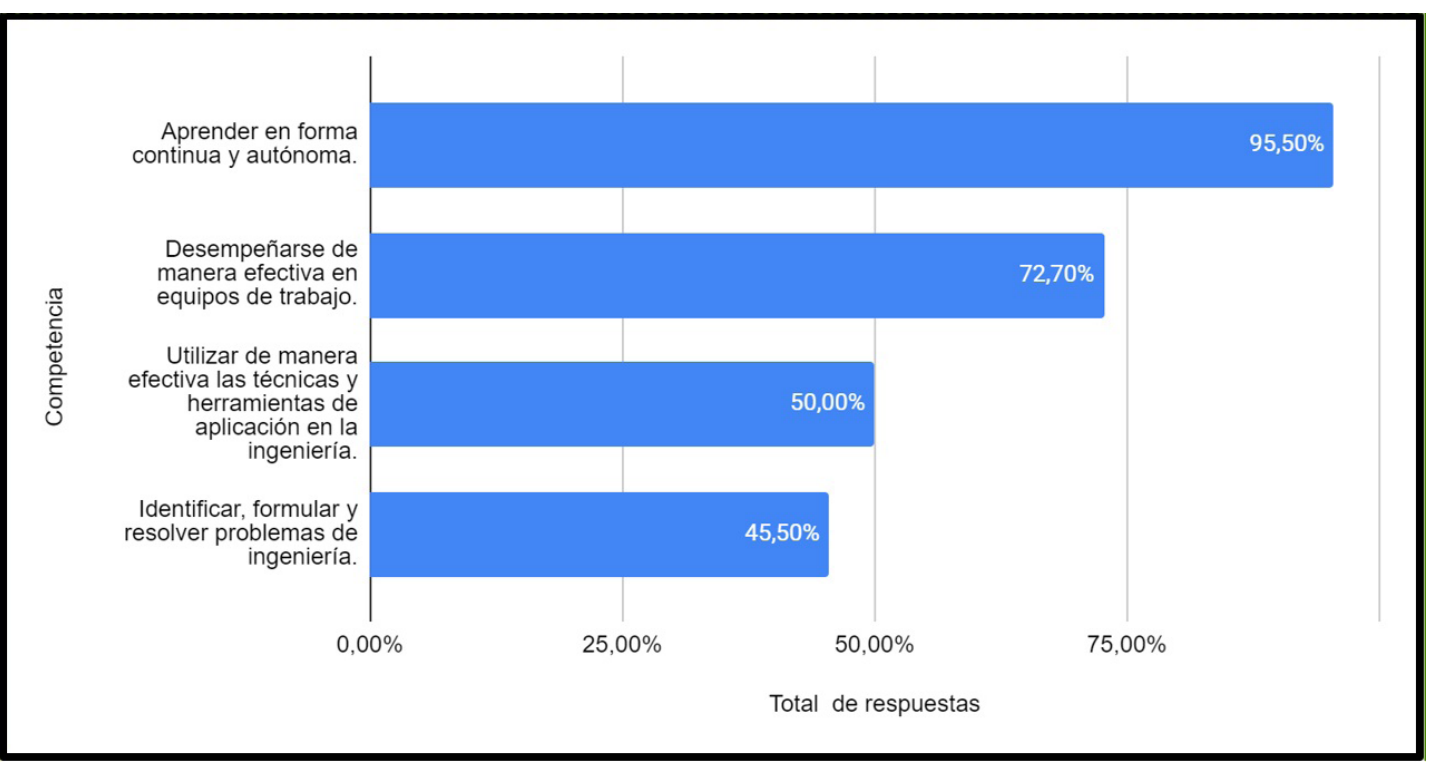

Gráfico Nº 10. Contribución del aula invertida a las competencias seleccionadas en el estudio-Docentes.

El mismo comportamiento se puede observar en el gráfico $\mathrm{N}^{\circ} 11$ que representa las opciones de los estudiantes.

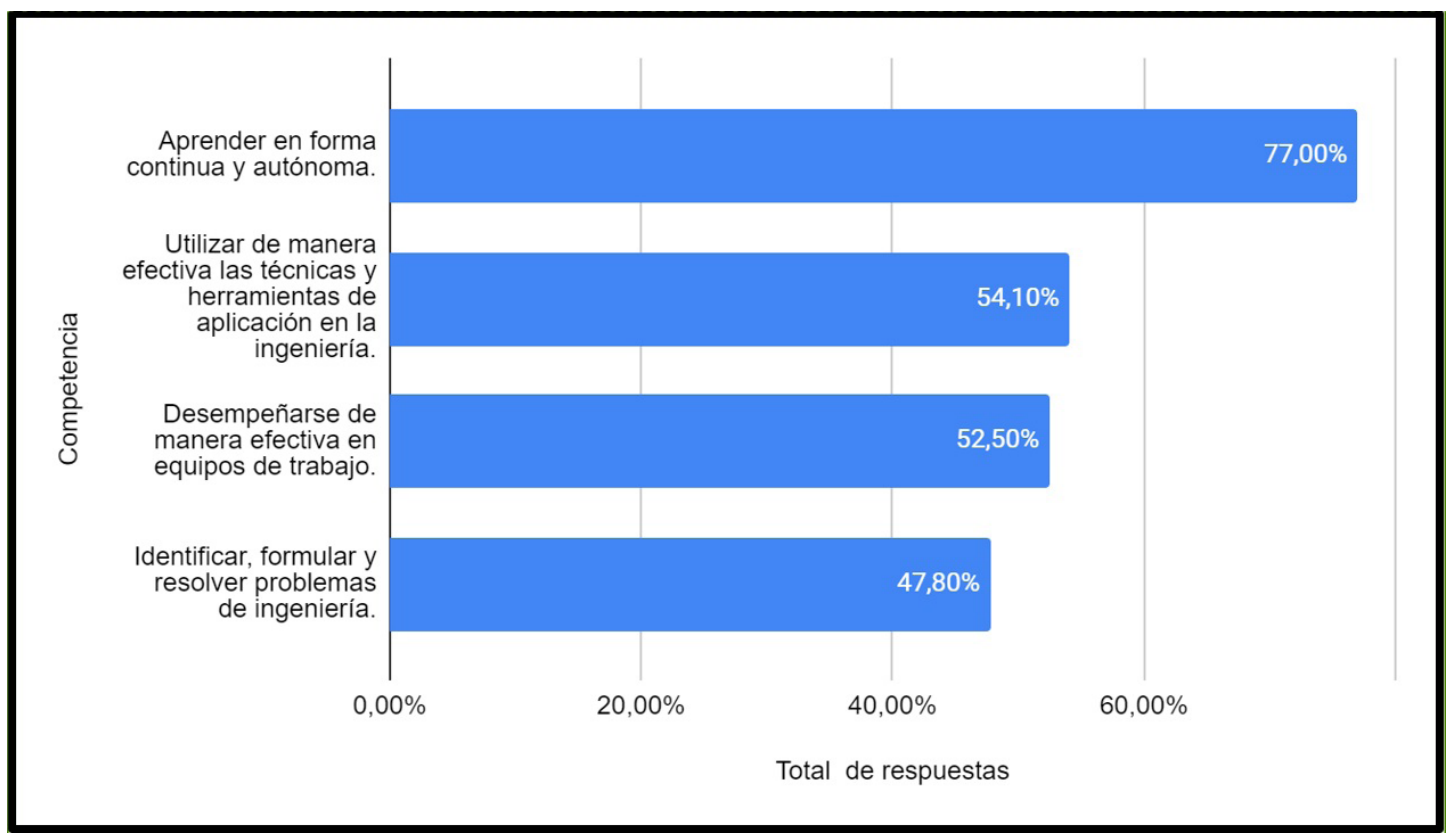

Gráfico № 11. Contribución del aula invertida a las competencias seleccionadas en el estudio- Alumnos. 
Con respecto a la implementación de la metodología de aula invertida en las aulas, un 50 \% contestó afirmativamente y el otro 50 \% “a veces”, según se muestran en el gráfico N N 12

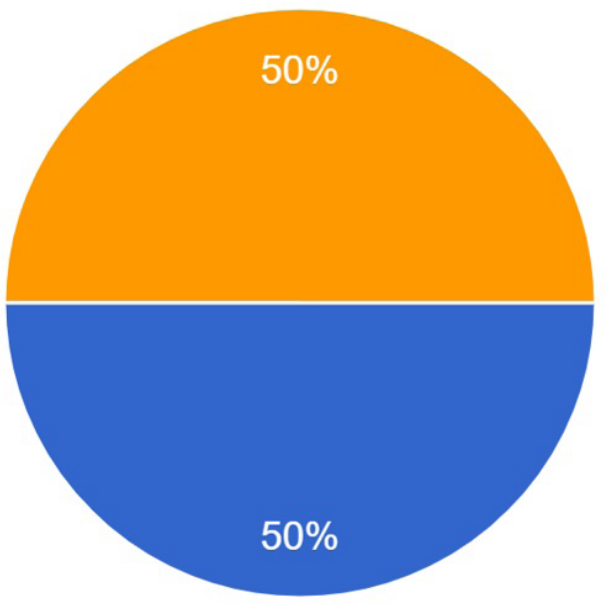

Sin embargo, cuando se preguntó a los alumnos si consideraban que hubiera algún profesor que haya utilizado la metodología de clase invertida en lo que va de su trayectoria en la carrera, la mayoría contestó afirmativamente. (Ver gráfico $\mathrm{N}^{\mathrm{o}} 13$ ).

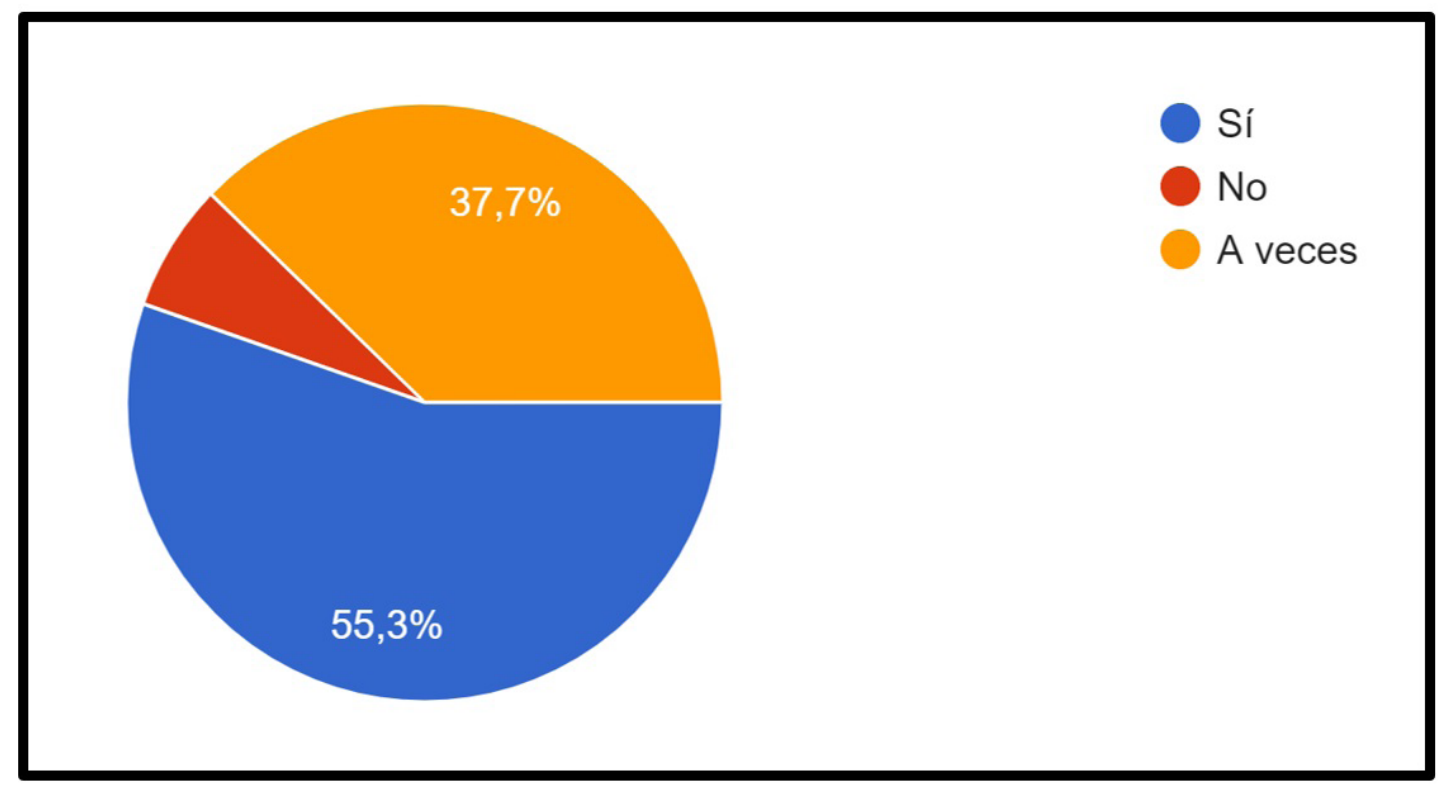

Gráfico N 13. Implementación de la metodología en el aula-Alumnos. 
Ante la pregunta, si le interesaría implementar la estrategia de aula invertida, el $100 \%$ de los docentes contestó afirmativamente (Ver gráfico $\left.\mathrm{N}^{\circ} 14\right)$.

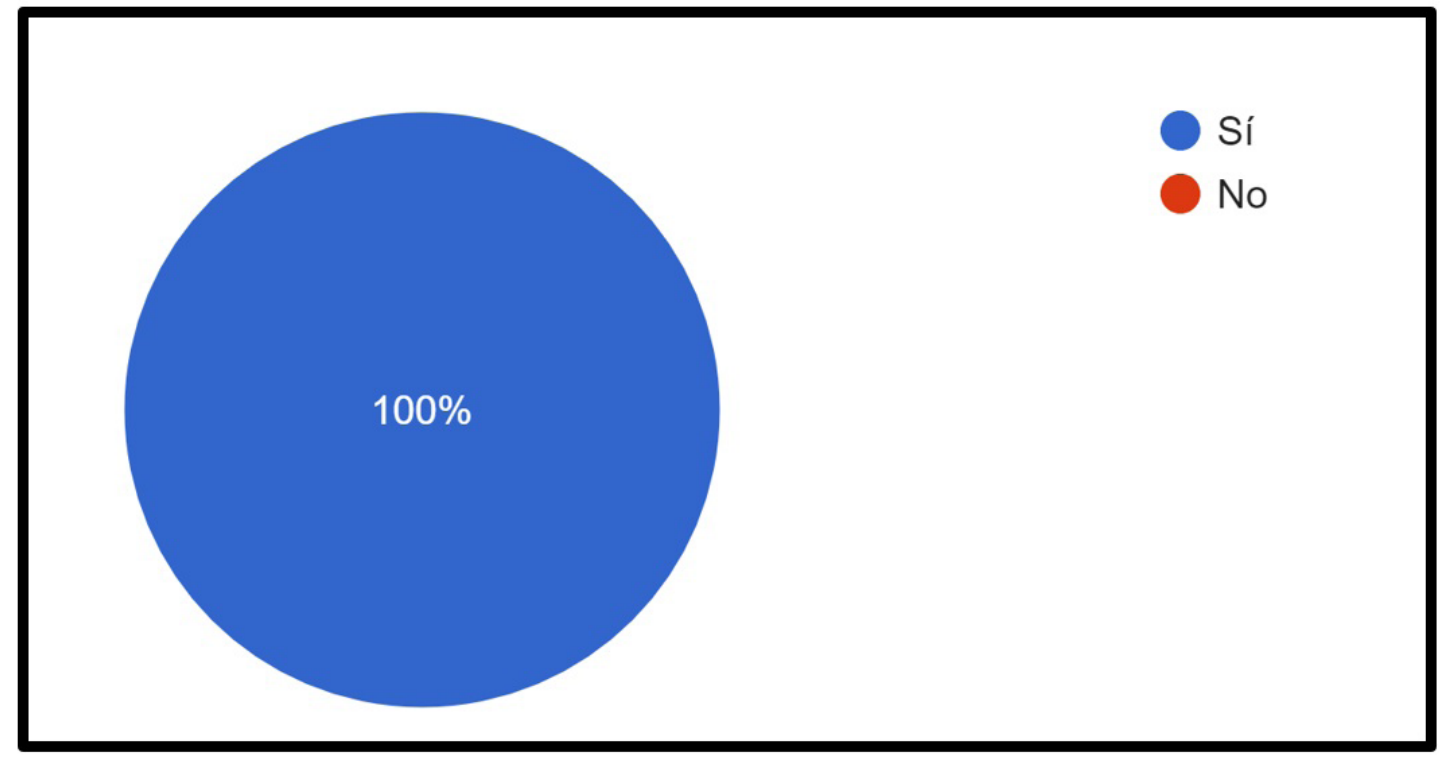

Gráfico Nº 14. Interés en la implementación de la metodología-Docente

Mientras que sólo un $73 \%$ de los alumnos contestaron afirmativamente (ver gráfico $\mathrm{N}^{\mathrm{o}}$ 15). Consideramos que esta respuesta está relacionada con la siguiente pregunta realizada a los alumnos.

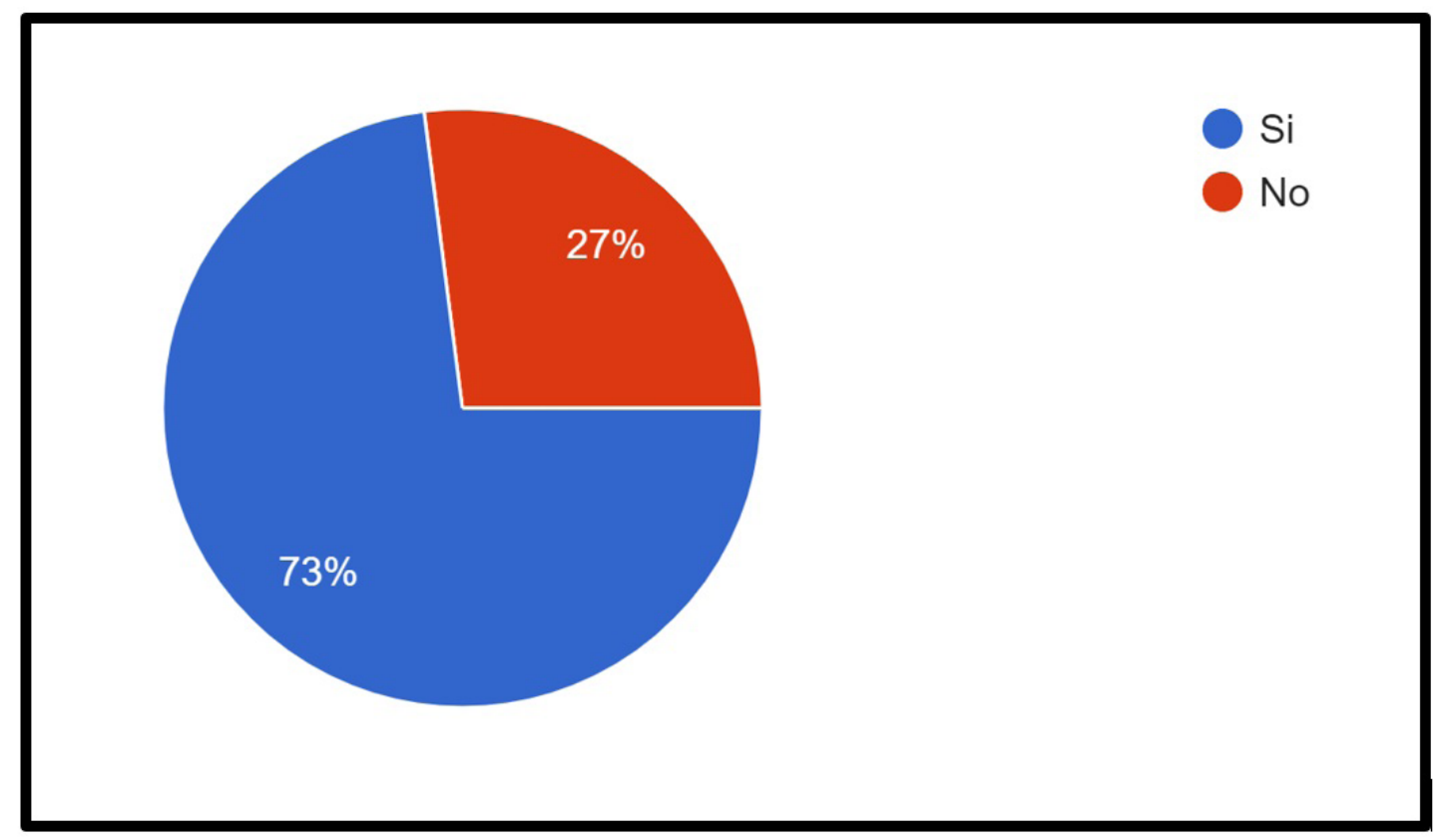

Gráfico N 15. Interés en la implementación de la metodología-Alumnos 
Ante la pregunta a los alumnos, sobre si la modalidad de aula invertida beneficia al alumno por sobre una clase magistral, siendo esta última el dictado en la que el docente es el protagonista de la enseñanza, es decir el profesor habla, el grupo de alumnos escucha, y ocasionalmente alguno de ellos interviene, preguntando o expresando algún comentario o alguna duda; el 56,6 \% contestó “depende” (ver gráfico $\mathrm{N}^{\circ} 16$ )

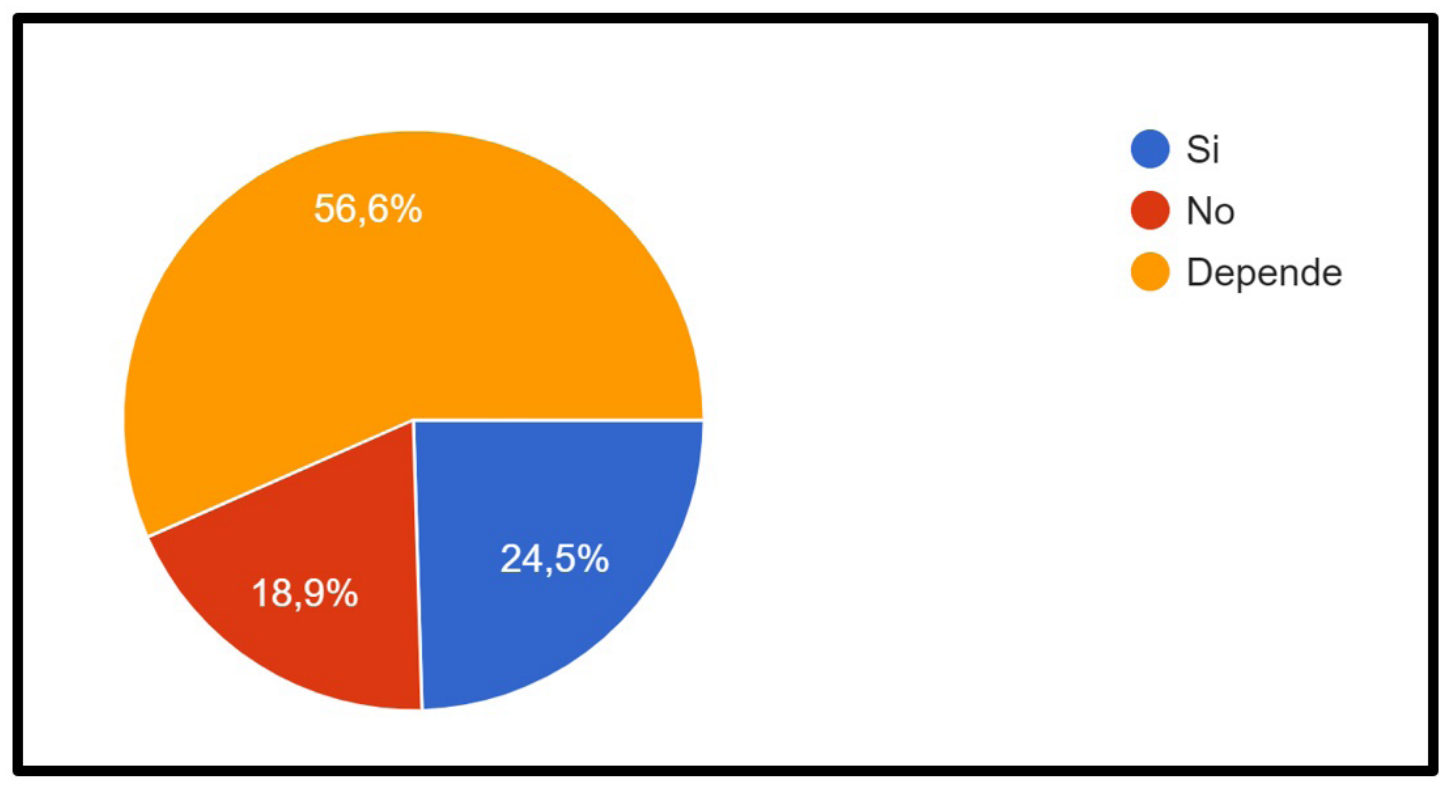

Gráfico N 16. Beneficio del aula invertida sobre clase magistral-Alumnos

Una respuesta representativa de la explicación a esas respuestas es la que se transcribe textualmente: "Creo que muchos profesores en lo que va del año hicieron un intento de implementación de aula invertida, pero solamente subieron vídeos sin dar clases y explicar bien los temas. Creo que hay que aclarar bien el concepto de esta modalidad, necesitamos que los docentes nos enseñen la base necesaria para poder investigar por nuestra cuenta después".

Se la considera beneficiosa a la metodología cuando: "el aprendizaje no se basa solo en el material sino en el aula se evacuan las dudas y complementa", "Cuando el alumno tiene los conocimientos o herramientas necesarias para resolver un problema", "Cuando investigamos anticipadamente el tema a tratar", "En aquellas materias donde el contenido es muy amplio para ser abarcado en la cursada y los temas tratados son actuales o en constante cambio. Promueve la investigación y estar al tanto de lo último, siempre que el alumno se sienta entusiasmado por el tema", "En los casos en que los profes son capacitados para aplicar de la manera correcta esta metodología”, etc.

Estos comentarios se relacionan directamente con las conclusiones del trabajo presentado por (Berenguer et al; 2016), citado en la introducción; en el mismo se plantea, que para la implementación de aula invertida es necesario un docente que actúe de guía en su proceso y que además de seleccionar cuidadosamente el material entregado a sus estudiantes mantenga una constante comunicación con ellos.

Cuando se preguntó a los docentes sobre la posibilidad de capacitarse para una mejor 
implementación de la metodología, como puede observarse en el gráfico $\mathrm{N}^{\circ} 17$; el 90, $9 \%$ considera pertinente una capacitación; mientras que un 9,1\% sólo tal vez.

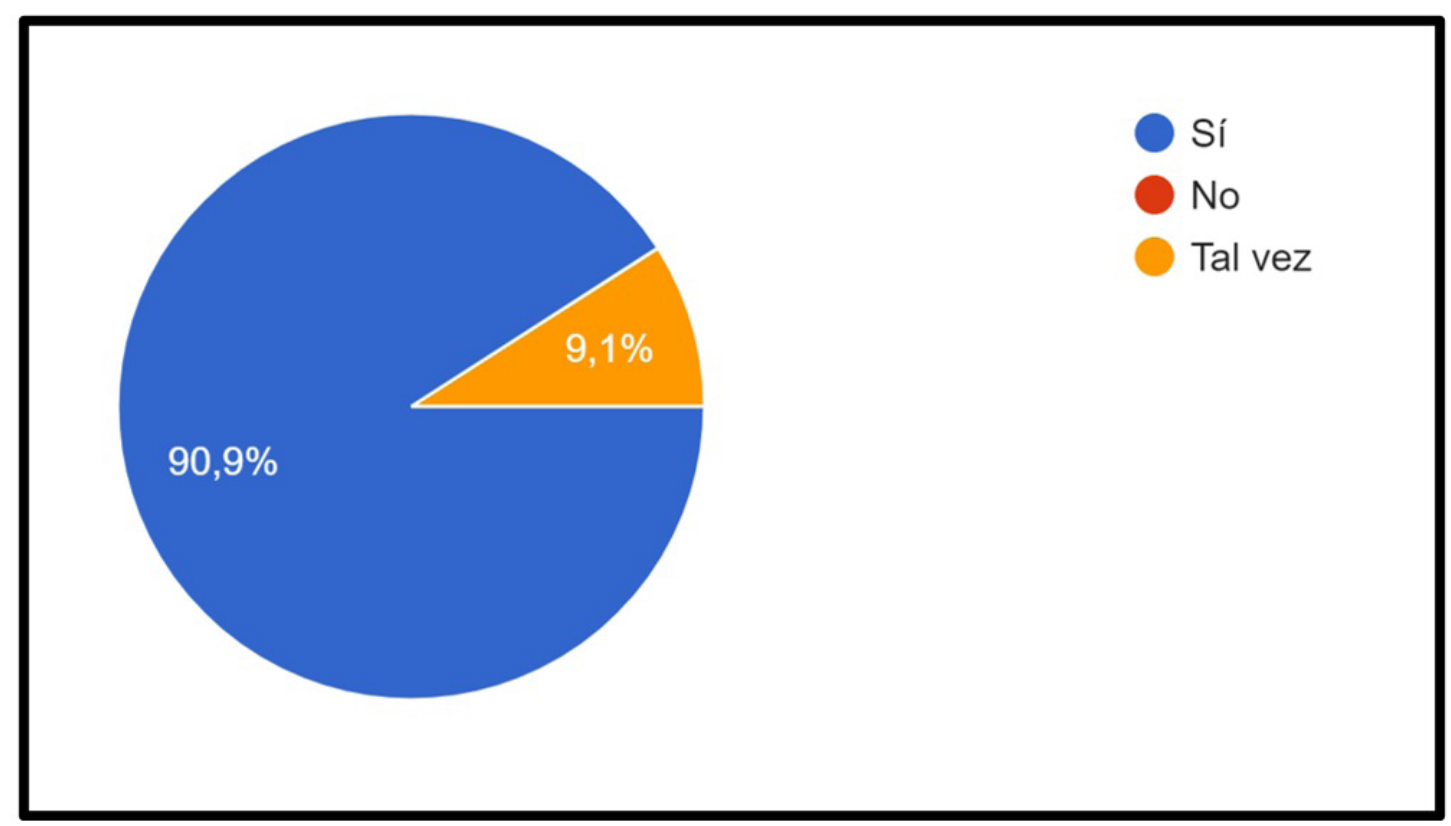

Gráfico N 17. Capacitación sobre herramientas para mejorar la implementación- Docentes.

Cabe aclarar, que los docentes que optaron por la opción "tal vez", refirieron en sus explicaciones a que estaba sujeto sus decisiones a la disponibilidad temporal y a las herramientas a trabajar en dicha capacitación.

Cuando se les preguntó a los docentes, sobre la herramientas utilizada para llevar adelante esta metodología, se mencionaron, entre otras, las siguientes: vídeos en youtube, ramificación, plataforma virtual, google drive, slack, blogs, aula virtual moodle con todas sus herramientas, videos de clase grabadas en años anteriores, videoconferencias, presentaciones, apuntes digitales, clases virtuales para atender consultas y facilitar los contenidos, sitios web de consulta, foros virtuales, documentos colaborativos, google forms, teams, wikis, blogs de los estudiantes como portfolio de autoevaluación, linkedin, office 365, google for education, poll everywhere, edmodo (microblogging educativo), suite google (buscador, planillas de cálculo, formularios, procesador de texto, drive), web meeting, pizarra digital, padlet, jitsi, hangouts, correo electrónico, whatsapp, trello y kahoot.

Mientras que los alumnos, manifestaron en un 72,3 \% que la herramienta más utilizada es el video. (ver gráfico $\mathrm{N}^{\circ} 18$ ) 


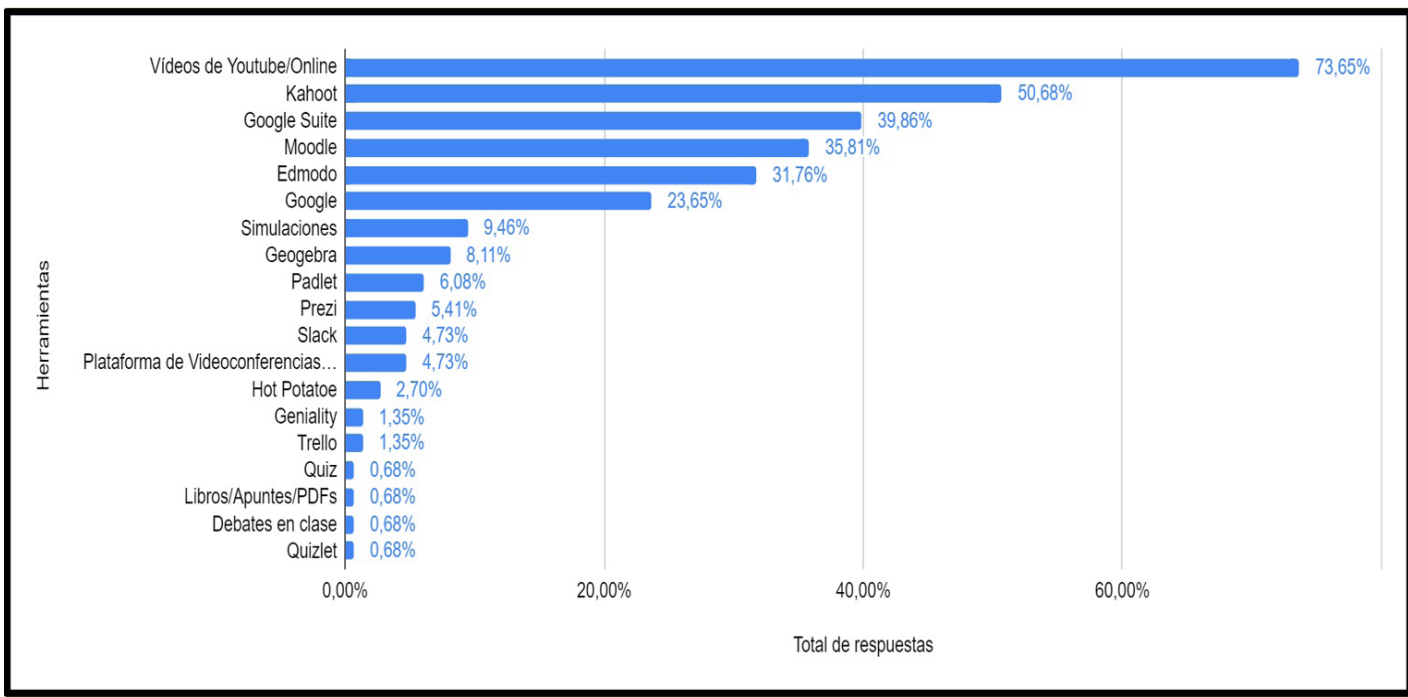

Gráfico N 18. Utilización de diferentes herramientas en el marco de la metodología - Alumnos

Estos datos coinciden con la mayoría de los estudios consultados, entre ellos el estudio de (Al Jassmi et al., 2019), sin embargo, es necesario tener en cuenta las conclusiones de este estudio en la que los autores manifiestan que los alumnos no estaban tan convencidos sobre la metodología al inicio del mismo; pero se adecuaron rápidamente a la misma. Los autores nos dejan como sugerencia que los docentes deberían tomarse 15 o 20 minutos de la clase antes de la resolución de los ejercicios para realizar una recopilación de los temas del video, con el fin de aclarar algunos conceptos que pudieran no haber quedado claros.

También se les preguntó a los alumnos si aquellos docentes que ellos consideran que aplicaron la metodología, la aplicaron bien, a los que un 55,4 \% contestó afirmativamente (ver gráfico $\mathrm{N}^{\circ} 19$ ); lo que nos muestra que los alumnos aplican un criterio de evaluación en relación con la implementación de la metodología. Este criterio podría ser coincidente con la apreciación de beneficios de la metodología.

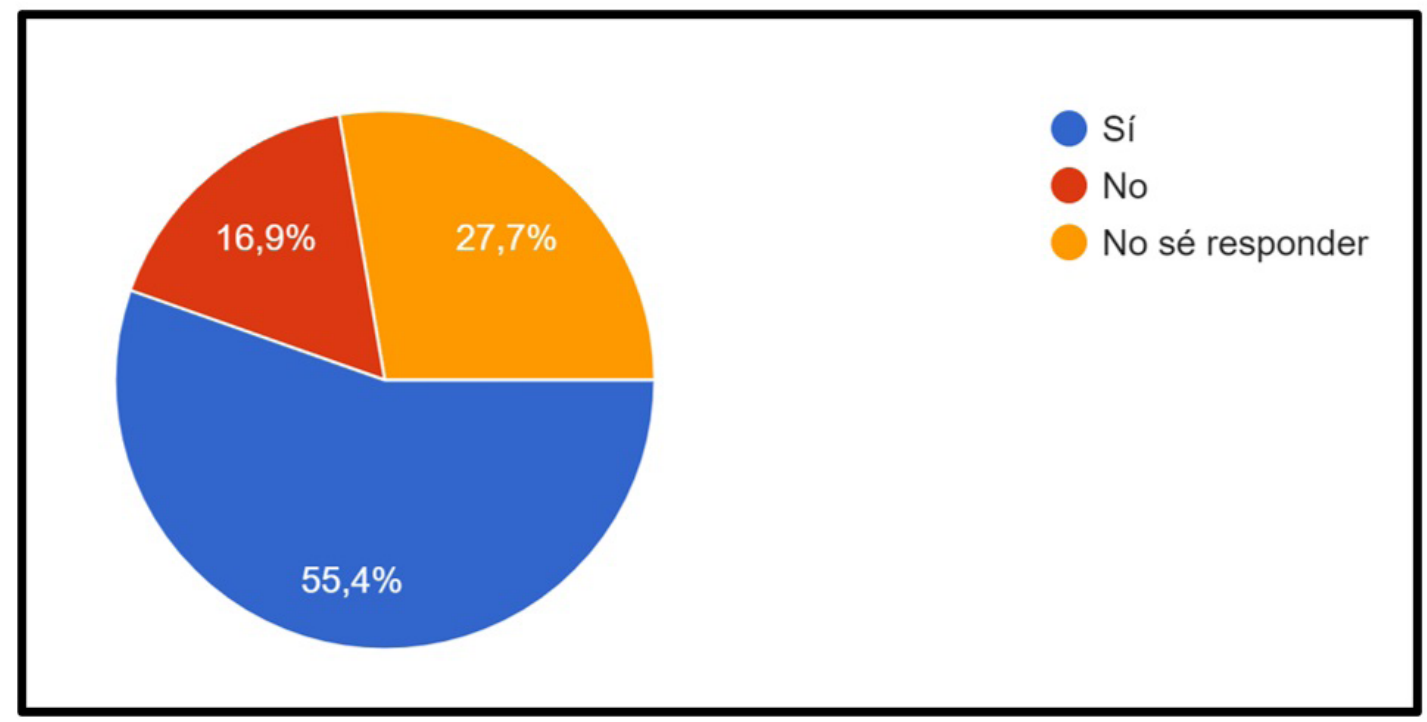

Gráfico N 19. Valoración positiva de la aplicación de la metodología - Alumnos 
Al respecto, se puede contrastar con las conclusiones del estudio de (Rodríguez, 2016), quien plantea, que si bien existe una gran aceptación por parte de los alumnos en la aplicación de la metodología; es relevante una correcta comunicación, la orientación del docente y fomentar el trabajo autónomo y colaborativo de los estudiantes.

\section{Conclusiones}

De los resultados obtenidos en las encuestas se desprenden las primeras conclusiones del estudio:

En general, el concepto de aula invertida es conocido por docentes y alumnos. Si bien se refieren a un tipo particular, entre las diferentes configuraciones, ambos actores están informados sobre la metodología. Sin embargo, debemos destacar que este aspecto de ninguna forma garantiza una implementación exitosa.

En cuanto a la contribución del aula invertida como estrategia adecuada para formar competencias ingenieriles, existe una disparidad en las opiniones. Se puede destacar aquí, que la postura de los docentes pareciera estar más a favor, comparativamente con la postura de los alumnos.

Como hipótesis que surge de este primer estudio, se podría aventurar que el estudiantado que se presenta pesimista ante la metodología opina de esa manera como consecuencia de las experiencias vividas en el transcurso de la carrera. En este sentido las recomendaciones realizadas por los estudios consultados en la revisión sistemática ayudarían en la capacitación a realizar con los docentes involucrados en la muestra.

Aguayo Vergara et al.,2019, señalan que si bien se utilizaron videos para implementar la metodología se observó que los profesores pueden adoptar paulatinamente nuevas tecnologías para proveer enseñanza asincrónica a los estudiantes, optimizando el trabajo en el aula con actividades de aplicación activa de los conceptos y el trabajo colaborativo. La implementación del aula invertida o flipped classroom implicó un cambio en la forma de planificar la enseñanza, los momentos para aprender, los roles en el proceso de enseñanza /aprendizaje. Es por ello, que es importante destacar y remarcar, que una estrategia de flipped classroom no debe estar basada única ni exclusivamente en videos, los cuales son una herramienta valiosa y poderosa, existiendo muchas otras que permiten invertir la clase según las necesidades de cada caso.

Un punto muy interesante a resaltar en las conclusiones es la predisposición de los docentes a realizar una capacitación para mejorar las estrategias que están implementando e incorporar nuevas herramientas del Web 3.0 que enriquezcan la metodología aula invertida.

Con relación a la generación de competencias, las apreciaciones positivas de los docentes son corroboradas con estudios como el de (Elmehdi et al.,2019), quienes además son coincidentes por otros citados por ellos. Entre sus aportes señalan "Nuestros resultados proporcionan evidencia concluyente de la clase invertida, el enfoque ha jugado un papel importante en la promoción de la participación de los alumnos y promovió interacciones entre pares, centró el aprendizaje en el alumno en lugar de ser controlado por el instructor. Para ser implementado con éxito, la institución necesita realizar talleres de capacitación y reestructurar el esquema del curso para garantizar que el contenido se cubra adecuadamente para garantizar el logro de los resultados de aprendizaje esperados.”

Con los datos recogidos en esta primera etapa se pretende implementar en el próximo año la capacitación a los docentes de las cátedras involucradas en la muestra y realizar un seguimiento de la implementación. 


\section{Referencias}

Aguayo Vergara, Margarita; Bravo Molina, Mario; Nocetti de la Barra, Alejandra; Concha Sarabia, Lucy; Aburto Godoy, Raquel (2019) Perspectiva estudiantil del modelo pedagógico flipped classroom o aula invertida en el aprendizaje del inglés como lengua extranjera. Revista Educación, vol. 43, núm. 1, Universidad de Costa Rica, Costa Rica

Al Jassmi, H., Ramesh, S., El-Maaddawy, T., Alqahtani, O., \& Ahmed, W. K. (2019). A Pilot Study on Civil Engineering Students Acceptance to a Flipped Classrooms Pedagogy. Advances in Science and Engineering Technology International Conferences (ASET), 1-4.

Bedoya, Ó. H., \& Hurtado Gil, S. V. (2019). Enseñanza de la ingeniería de software basada en competencias fundamentales y aula invertida. $2^{\circ}$ Congreso Latinoamericano de Ingeniería - Encuentro Internacional de Educación en Ingeniería ACOFI. Cartagena de Indias.

Berenguer-Albaladejo, C. (2016). Acerca de la utilidad del aula invertida o flipped classroom. XIV Jornadas de Redes de Investigación en Docencia Universitaria: investigación, innovación y enseñanza universitaria: enfoques pluridisciplinares, (págs. 1466-1480). Alicante

Castells, M. (2014). El impacto de internet en la sociedad: una perspectiva global. C@ mbio, 19, 127-147.

Elmehdi, H. M., Dalah, E. Z., \& Ibrahem, A. M. (2019, March). Impact of Flipped Physics Classes on the Performance of Engineering Students: University of Sharjah Case Study. In 2019 Advances in Science and Engineering Technology International Conferences (ASET) (pp. 1-6). IEEE.

Ponce, S. L., Marichal, A., Martínez, G., Soldini, M., \& Ponce, R. D. (2017). Implementación de la clase invertida en el aula universitaria: posibilidades para la obtención de aprendizaje no superficiales. XII Congreso de Tecnología en Educación y Educación en Tecnología TEyET. La Matanza: Universidad Nacional de La Matanza - REd UNCI.

Rodríguez, W. P. (2016). Estudio de Evidencias de aprendizaje significativo en un aula bajo el modelo flipped classroom. Revista Electrónica de Tecnología Educativa - EDUTEC.

Xie, Y. M. (14 de marzo de 2019). Yi Miu Shum Xie - Social Media, Marketing, SEO, Marca Personal. Obtenido de https://yiminshum.com/digital-social-media-argentina-2019/ 